\title{
Miranda
}

Revue pluridisciplinaire du monde anglophone /

Multidisciplinary peer-reviewed journal on the English-

speaking world

1 | 2010

Variations on Darwin

\section{Darwin, les fossiles et les bases de la classification moderne}

Francis Duranthon

URL : http://journals.openedition.org/miranda/533

DOI : $10.4000 /$ miranda.533

ISSN : 2108-6559

Éditeur

Université Toulouse - Jean Jaurès

\section{Référence électronique}

Francis Duranthon, «Darwin, les fossiles et les bases de la classification moderne », Miranda [En ligne] 1 | 2010, mis en ligne le 23 mars 2010, consulté le 16 février 2021. URL : http://

journals.openedition.org/miranda/533; DOI : https://doi.org/10.4000/miranda.533

Ce document a été généré automatiquement le 16 février 2021.

\section{(c) (i) () $\Theta$}

Miranda is licensed under a Creative Commons Attribution-NonCommercial-NoDerivatives 4.0 International License. 


\title{
Darwin, les fossiles et les bases de la classification moderne
}

\author{
Francis Duranthon
}

1 Le besoin de classer les espèces semble inhérent à la nature humaine. Toutes les civilisations, toutes les cultures humaines ont adopté des classifications, depuis des temps immémoriaux. En effet, c'est en classant et en nommant les organismes animaux et végétaux que l'homme a pu et peut encore décrire le monde qui l'entoure et se l'approprier à des fins utilitaires ou même commerciales de nos jours (c'est ainsi que l'on en arrive à breveter le génome de certaines espèces).

\section{Antiquité et Moyen-Âge}

2 Au cours de l'histoire, de nombreux systèmes classificatoires ont vu le jour. C'est surtout dans le monde végétal qu'ils se sont développés. Dès l'antiquité égyptienne, vers 1600 av. JC, le papyrus Ebers recense un certain nombre de plantes et les classe selon leurs propriétés médicinales. Le traité apocryphe d'Aristote sur les plantes, vers 325 av. JC, rédigé par son disciple Theophraste, classe les arbres et les arbustes selon leur taille. Ce dernier, parfois considéré comme le père de la Botanique, développe d'ailleurs une classification des plantes d'après leur usage médicinal dans le Traité des Plantes. $\mathrm{Au} \mathrm{I}^{\mathrm{er}}$ siècle après $\mathrm{JC}$, Dioscoride distingue les plantes alimentaires, les aromatiques, les médicinales et les vénéneuses dans son ouvrage Materia medica. Même si le Moyen Age fut une période relative d'obscurantisme dans le domaine scientifique, on peut citer les travaux d'Albert le Grand (1193-1280), qui fut le professeur de Saint Thomas d'Aquin. Il parvint à différencier les plantes à partir de la structure de la tige dans son ouvrage De vegetalis. Pour lui, la fonction d'un organe détermine sa forme et il distingue ainsi les plantes dicotylédones des monocotylédones. La Renaissance connaît un profond renouvellement des travaux soutenus par les premières grandes expéditions maritimes de découverte et les tentatives d'acclimatation des différentes espèces végétales ramenées par les explorateurs. 


\section{Renaissance}

3 Conrad Gesner (1516-1565), éminent naturaliste suisse, élabore une classification botanique fondée sur les fleurs et les fruits. Parallèlement, il commence à publier dès 1551 son Historia animalium, dont le dernier volume sera publié 22 ans après sa mort. Dans cet ouvrage immense de 3500 pages, chaque espèce est minutieusement décrite. Gessner mentionne le nom de chaque espèce dans plusieurs langues (vivantes ou mortes), son habitat, son origine, sa description anatomique, sa physiologie, les qualités de son âme, les divers usages que l'on peut en tirer, son intérêt alimentaire et médical, ainsi que son utilisation par les poètes et les philosophes. La classification qu'il utilise reprend pour l'essentiel les catégories populaires : quadrupèdes ovipares et vivipares, oiseaux, poissons... Gessner, confronté à l'impossibilité d'établir une classification cohérente, opte pour un ordre alphabétique. Il propose, cependant, une taxinomie embryonnaire, et emploie une appellation latine de deux mots: le premier, le genre, suivi d'un qualificatif - système qui sera plus tard suivi par d'autres naturalistes comme Gaspard Bauhin, John Ray, et surtout Carl von Linné.

Gaspard Bauhin (1560-1624) écrit un traité de la botanique descriptive, Pinax Theatri Botanici, publié à Bâle en 1671, quoique sa rédaction date de 1596. Il y recherche les ressemblances et les différences existant chez les végétaux. Il reprend à son compte la systématique binominale de Gessner mais rompt avec l'ordre alphabétique en plaçant, par exemple, avec justesse la pomme de terre dans la famille des Solanacées. De son côté, Andrea Cesalpino (1519-1603) développe la première classification méthodique des végétaux dans son De plantis libri en 1583 en se fondant sur la forme de la fleur, du fruit et sur le nombre des graines.

5 En 1686, John Ray tente une première classification naturelle des plantes et expose sa méthode dans trois ouvrages: Methodus plantarum nova (1682), le premier volume d'Historia plantarum (1686), et dans Methodus emendata (1703). Il est à l'origine de la classification naturelle utilisant plusieurs caractères. Il sépare ainsi nettement les monocotylédones des dicotylédones, les gymnospermes des angiospermes, et écarte aussi les plantes sans fleurs (comme les fougères) des plantes à fleurs.

6 Joseph Pitton de Tournefort, quant à lui, propose en 1694 une méthode pour reconnaître les plantes et l'aspect des fleurs, d'après la constitution de la corolle. Il reprend à son compte la notion de genre, proposée près de 150 ans auparavant par Conrad Gesner.

\section{Les Lumières}

C'est au XVIII ${ }^{\mathrm{e}}$ siècle que les progrès sont les plus considérables et vont marquer durablement la classification. Un personnage majeur de cette époque est assurément le botaniste suédois Carl von Linné (1707-1778). Linné établit une classification selon le sexe des plantes, d'après les étamines, et s'attache à la notion d'espèce. Ses ouvrages, Genera plantarum (1737) et Species Plantarum (1753), restent à la base des classifications. Mais c'est avec la dixième édition de son Systema Naturae (1758) qu'il donne la classification des végétaux, animaux et minéraux et généralise à tous les organismes l'utilisation de la nomenclature binominale amorcée dans les travaux de Gessner et de John Ray. C'est la nomenclature binaire latine : un nom de genre, un nom d'espèce. Les 
espèces y sont regroupées de manière hiérarchisée, au sein de genres, eux-mêmes rassemblés en ordres, et les ordres en classes. Dans la vision de Linné, le concept de famille, niveau intermédiaire entre le genre et l'ordre, pourtant déjà élaboré par le botaniste français Pierre Magnol dans Prodromus historice generalis plantarum, in quo familice per tabulas disponuntur (1689), n'existe pas. C'est à partir de cette date fondatrice que tous les naturalistes suivront cette manière de nommer les espèces et de classer. On parle dès lors de classification linnéenne. A travers ce mode de classification, les auteurs recherchent une classification dite naturelle, représentant un ordre de la Nature, parfaitement compatible avec la pensée judéo-chrétienne et les opinions de l'Eglise et la Genèse. Dans cette vision, l'homme se trouve au sommet de la hiérarchie, au sein d'un groupe appelé les Primates (les premiers).

8 Avant la Révolution, Bernard de Jussieu (1699-1777) et son neveu Antoine-Laurent (1748-1836) amènent une nouvelle notion fondamentale: la subordination des caractères, publiée par Antoine-Laurent en 1789 dans Genera plantarum. En biologie, un caractère correspond à tout attribut observable d'un organisme : il peut être génétique, anatomique, biochimique, caryologique, éthologique ou encore embryologique. Les Jussieu se rendent compte que, pour déterminer un groupe donné, il est nécessaire d'identifier un (ou plusieurs) caractère(s) constant(s) à l'intérieur du groupe et variable(s) dans tous les autres. Un caractère, comme la possession de vertèbres par exemple est donc utile à un niveau précis de la classification, pour définir une classe, celle des vertébrés. Par contre, la forme des vertèbres servira à définir les niveaux inférieurs de la classification. Les caractères sont donc "subordonnés" les uns aux autres et, dès lors, il faut trier et hiérarchiser les caractères pour utiliser ceux qui sont discriminants à un niveau donné pour la pratique classificatoire. A partir d'observations très fines, ils proposent ainsi un classement hiérarchisé des caractères des végétaux : étamines, pistil, fleur. Cette méthode et ses résultats, adoptés par l'ensemble des botanistes, signe la fin de la classification de Carl von Linné sur les organes sexuels. Cependant la nomenclature binominale, c'est-à-dire la manière de nommer les espèces avec deux noms latins (genre, espèce) demeure et est encore utilisée de nos jours.

Ce principe de subordination des caractères est repris par les zoologistes au début du $\mathrm{XIX}^{\mathrm{e}}$ siècle. Georges Cuvier (1769-1832) développe de son côté la notion de plans d'organisation et définit 4 embranchements (les vertébrés (Vertebrata), les articulés (Articulata), les mollusques (Mollusca) et les radiaires (Radiata)), caractérisés par des dispositions très différentes des organes les uns par rapport aux autres (Cuvier 1812).

\section{Les fossiles et l'idée d'évolution}

Parallèlement, depuis des temps immémoriaux, les fossiles ont intrigué les hommes. Les Magdaléniens par exemple les ramassent, et on retrouve ainsi des ammonites au sein des couches archéologiques de cette époque. Aristote considère que les fossiles ont été façonnés directement dans la boue, à partir d'une force formatrice mystérieuse. Pour Léonard de Vinci (1452-1519), qui s'étonne de découvrir des restes d'organismes marins au cœur des terres, « ce qui était jadis le fond de la mer est devenu le sommet des montagnes", et il pense que "sur les plaines d'Italie au-dessus desquelles volent aujourd'hui des oiseaux, de larges bancs de poissons se sont un jour déplacés » (Vinci, 1508-10). Bernard Palissy (1510-1589/90) s'interroge : «Pourquoi trouve-t-on tant de 
fragments de coquilles entre deux couches de pierres, sinon parce que ces coquilles déjà déposées sur la plage y furent recouvertes d'une terre rejetée par la mer, laquelle terre est venue ensuite à se pétrifier ?» (Palissy, 1563).

Une autre question agite fortement les savants jusqu'à la Renaissance, celle de la nature des glossopètres, ces langues de pierre qui sont en réalité, comme le démontrera Fabio Colonna (1616), des dents de requins. Pour Pline l'ancien (23-79) :

La glossopètre, semblable à la langue de l'homme, ne s'engendre point, dit-on, dans la terre, mais tombe du ciel pendant les éclipses de lune; elle est nécessaire à la sélénomantie ; mais nous avons été rendus incrédules par la vanité d'une promesse comme celle-ci, à savoir que cette pierre fait cesser les vents. (Pline l'ancien T2, livre XxxvII, 563)

12 Grâce à Colonna, la nature animale des fossiles est comprise, et Nicolas Stenon (1638-1686) conclura que les fossiles peuvent changer de composition, sans que cela soit visible extérieurement. Leur origine animale est démontrée. Mais le problème reste entier. Que représentent ces organismes ? Comment les interpréter dans le cadre défini par la religion chrétienne? Sont-ils les témoins du déluge? C'est ce qui conduira, par exemple, Johann Jakob Scheuchzer (1672-1733) à considérer comme «Homo diluvii testis» (homme preuve du déluge) un reste de salamandre fossile dans son ouvrage Physica sacra (1731). Il faudra attendre le début du XIX ${ }^{\mathrm{e}}$ siècle pour que les conceptions évoluent de manière sensible.

13 En 1809, Jean-Baptiste Lamarck, dans sa Philosophie zoologique, fait émerger la notion de transformisme, c'est-à-dire l'idée que les espèces se transforment et se modifient au cours du temps en se devenant plus complexes.

Comment, en effet, pouvois-je envisager la dégradation singulière qui se trouve dans la composition de l'organisation des animaux, à mesure que l'on parcourt leur série, depuis les plus parfaits d'entr'eux, jusques aux plus imparfaits, sans rechercher à quoi peut tenir un fait si positif et aussi remarquable, un fait qui m'est attesté par tant de preuves? Ne devois-je pas penser que la nature avoit produit successivement les différens corps doués de la vie, en procédant du plus simple vers le plus composé; puis qu'en remontant l'échelle animale depuis les animaux les plus imparfaits jusqu'aux plus parfaits, l'organisation se compose et même se complique graduellement, dans sa composition, d'une manière extrêmement remarquable? (Lamarck ii-iii).

14 Si le rôle du temps est sous-jacent dans cet extrait, il est beaucoup plus net dans le passage qu'il consacre aux fossiles :

Or, si quantité, de ces coquilles fossiles se montrent avec des différences qui ne nous permettent pas, d'après les opinions admises, de les regarder comme des analogues des espèces avoisinantes que nous connoissons, s'ensuit-il nécessairement que ces coquilles appartiennent à des espèces réellement perdues? Pourquoi, d'ailleurs, seroient-elles perdues, dès que l'homme n'a pu opérer leur destruction? Ne seroit-il pas possible, au contraire, que les individus fossiles dont il s'agit appartinssent à des espèces encore existantes, mais qui ont changé depuis, et ont donné lieu aux espèces actuellement vivantes que nous en trouvons voisines. Les considérations qui suivent, et nos observations dans le cours de cet ouvrage, rendront cette présomption très probable. Tout homme observateur et instruit sait que rien n'est constamment dans le même état à la surface du globe terrestre. Tout, avec le temps, y subit des mutations diverses plus ou moins promptes, selon la nature des objets et des circonstances (Lamarck 77-8).

15 Cette notion du rôle du temps est essentielle. Elle apparaît déjà de manière non explicite dans les travaux de Robert Hooke (1635-1703) qui, dans son ouvrage, Lectures 
and Discourses of Earthquakes and Subterraneous Eruptions (1668), considère que les fossiles témoignent de changements dans les organismes de la planète et que des espèces sont apparues et ont disparu. Mais c'est avec Buffon (1749) que les outils intellectuels rompent enfin avec le dogme chrétien. Buffon étudie le temps de refroidissement d'un boulet de canon et en applique ses résultats à la terre, qui, selon lui, a été à un moment une masse en fusion. Il montre ainsi que l'âge de 6000 ans, calculé par les théologiens pour la formation de la planète à partir du recensement des générations décrites dans la Bible, est bien inférieur à la réalité. Parallèlement, à partir de l'étude de restes de proboscidiens (groupe d'animaux auquel appartient l'éléphant) fossiles d'Amérique du Nord, il crée avec son alter ego Daubenton le concept d'espèces perdues (Tassy, 2002), auquel fait allusion Lamarck dans l'extrait ci-dessus, ouvrant ainsi la voie au développement de la paléontologie. Peu de temps après, Georges Cuvier, qui fonde la paléontologie des vertébrés, se rend bien compte que les couches fossilifères contiennent des organismes disparus, dont on ne connaît pas d'équivalent sur la planète. Mais il ne trouve pas de faunes intermédiaires entre une couche géologique et celle qui lui est immédiatement supérieure. Comme il ne croit pas en l'évolution des espèces (on le qualifie aujourd'hui de " fixiste »), il développe alors dans Discours sur les Révolutions du globe (1825) l'idée de créations successives avec des renouvellements fauniques complets, qui lui permettent ainsi de mettre en phase ses observations avec la durée des temps géologiques. Cette manière de voir les choses permettra ainsi d'ouvrir la voie à une nouvelle discipline, la stratigraphie, dont Alcide d'Orbigny (1802-1857) se fera le chantre :

Les animaux ne montrant dans leurs formes spécifiques aucune transition, se sont succédé [sic] à la surface du globe, non par passage, mais par extinction des races existantes et par la création successive des espèces à chaque époque géologique (D'Orbigny 1840, 156) .

D'Orbigny, qui n'accepte pas lui non plus l'idée d'évolution, va ainsi définir 28 étages géologiques, du Silurien à l'actuel. Pour lui, chaque étage a ses fossiles et chaque fossile correspond à un étage et un seul :

Si nous trouvions dans la nature des formes qui, après l'analyse la plus scrupuleuse, ne nous offriraient encore aucune différence appréciable, quoiqu'elles fussent séparées par un intervalle de quelques étages (ce qui n'existe pas encore), nous ne balancerions pas un instant à les regarder néanmoins comme distinctes. Lorsqu'on voit toutes les formes spécifiques bien arrêtées avoir des limites fixes dans les étages, et appartenir à un seul, on doit croire que ce sont nos moyens de distinction qui sont insuffisants pour trouver les différences entre ces deux espèces d'époques éloignées qui se ressemblent (D'Orbigny 1850-52, xxxvIII).

17 Cuvier, fixiste, nie par ailleurs l'idée de Lamarck que l'on puisse classer les espèces par complexité ou perfection croissante en faisant paradoxalement référence à ce que nous nommons aujourd'hui l'évolution buissonnante :

Sans doute, il y a, chez les Mollusques, une série de dégradation d'un plan commun tout aussi suivie que parmi les animaux vertébrés, en sorte que l'on peut descendre de la seiche jusqu'à l'huître à peu près comme de l'homme jusqu'à la carpe ; mais ce n'est point par une seule ligne que l'on descend ainsi dans un embranchement, pas plus que dans l'autre. (Cuvier 1812, 79)

18 Étienne Geoffroy Saint-Hilaire (1772-1844) définit ensuite, sans la nommer ainsi, la notion d'homologie qui permet de comparer des organismes ayant le même plan d'organisation (Saint-Hilaire, 1822). Deux organes sont homologues lorsqu'ils ont une même situation dans un plan d'organisation. Plus tard, il sera démontré qu'ils 
témoignent d'une même origine embryologique et de l'existence d'un ancêtre commun. Cette notion va à l'encontre de l'idée de similitude globale. En effet, deux organes peuvent être homologues et n'avoir ni même taille, ni même forme, ni même fonction. Les pattes antérieures des mammifères en sont un bon exemple: la patte du cheval, l'aile de la chauve-souris, l'aileron de la baleine sont homologues à l'échelle des vertébrés des tétrapodes.

Né en 1809, l'année même où Lamarck publie sa Philosophie zoologique, Charles Darwin a d'abord été voyageur-naturaliste. Après son tour du monde sur le Beagle, il a passé des années à décrire, nommer et classer de petits crustacés : les cirripèdes. En 1859, avec l'Origine des espèces, il propose, en intégrant une quantité impressionnante de faits d'ordre zoologique et botanique, une théorie qu'il appelle «descendance avec modification » et que nous appelons aujourd'hui « évolution ». Pour lui, les caractères utiles pour la classification sont ceux qui sont hérités d'un ancêtre commun; ainsi, il affirme "Our classifications will come to be, as far as they can be so made, genealogies» (Darwin 1859, 486). Pour lui, la communauté de descendance est le lien caché que les naturalistes ont vainement cherché depuis le XVIII ${ }^{\mathrm{e}}$ siècle. Il faut mettre en relation les organismes fossiles avec les espèces actuelles. Classer les animaux et proposer une phylogénie (terme défini par le biologiste allemand Ernst Hæckel) ne sont qu'une seule et même pratique. Comme il n'y a qu'une seule histoire de la vie sur terre, il n'existe et ne peut exister qu'une seule classification naturelle, celle qui intègre la généalogie des espèces. Il est d'ailleurs assez révélateur que la seule figure de son ouvrage soit consacrée à un modèle théorique de ce que nous nommerions aujourd'hui un arbre phylogénétique. Toutefois, dans cette figure, il ne mentionne pas d'échelle de temps, mais indique seulement les niveaux de spéciation par des lignes notées I, II, III... Car Darwin est conscient que les plus grosses objections que l'on peut faire à sa théorie concernent l'absence de fossiles intermédiaires, notamment au niveaux supérieurs de la classification - les fameux " chaînons manquants » :

Lastly, looking not to any one time, but to all time, if my theory be true, numberless intermediate varieties, linking most closely all the species of the same group together, must assuredly have existed; but the very process of natural selection constantly tends, as has been so often remarked, to exterminate the parent forms and the intermediate links. Consequently evidence of their former existence could be found only amongst fossil remains, which are preserved, as we shall in a future chapter attempt to show, in an extremely imperfect and intermittent record. (Darwin 1866, 179)

Et il conclut :

Geology assuredly does not reveal any such finely graduated organic chain; and this, perhaps, is the most obvious and gravest objection which can be urged against my theory. The explanation lies, as I believe, in the extreme imperfection of the geological record. (Darwin 1859, 298)

21 Pourtant, rapidement, la découverte d'un fossile extraordinaire, à Solnhofen en Allemagne, va donner raison à sa théorie. Ce fossile, l'Archcoopteryx, est signalé par le paléontologue allemand Herman von Meyer (1861). Acheté deux ans plus tard par le Natural History Museum de Londres, il est étudié et décrit par le paléontologue britannique Richard Owen (1863).

Très rapidement, la controverse naît autour de cet animal. Pour certains, il s'agit d'un reptile ailé, pour d'autre, Archceopteryx est clairement un oiseau. Il faut dire que l'animal est singulier. Sa mandibule porte clairement de petites dents pointues, des 
doigts griffus et il est muni d'une longue queue comprenant de nombreuses vertèbres. Ce sont typiquement des caractères de reptiles. Par contre, il possède également des caractères d'oiseaux : des plumes et un os en forme d'Y qui résulte de la fusion des deux clavicules. C'est un peu comme s'il s'agissait d'une sorte d'hybride monstrueux. Archoeopteryx apparait alors comme le chaînon manquant, l'intermédiaire rêvé entre les reptiles et les oiseaux et il arrive à point pour confirmer les théories évolutionnistes de Darwin qui déclare dans la quatrième édition de son ouvrage :

And still more recently, that strange bird, the Archeopteryx [sic], with a long lizardlike tail, bearing a pair of feathers on each joint, and with its wings furnished with two free claws, has been discovered in the oolitic slates of Solnhofen. Hardly any recent discovery shows more forcibly than this how little we as yet know of the former inhabitants of the world. (Darwin 1866, 399)

Cet animal, au point de vue de la classification, représente le parfait intermédiaire entre la classe des Reptiles et celle des Oiseaux. Thomas Huxley (1825-1895), farouche défenseur des idées évolutionnistes (au point d'être surnommé le bouledogue de Darwin), conclura même à une origine dinosaurienne des oiseaux en s'appuyant sur ce fossile (Huxley, 1868). Darwin reprendra à son compte ses conclusions dans la cinquième édition de son ouvrage :

Even the wide interval between birds and reptiles has been shown by Professor Huxley to be partially bridged over in the most unexpected manner, by, on the one hand, the ostrich and extinct Archeopteryx [sic], and on the other hand, the Campsognathus [sic], one of the Dinosaurians - that group which includes the most gigantic of all terrestrial reptiles. (Darwin 1869, 402-3)

Si cette hypothèse d'étroite parenté entre dinosaures et oiseaux a été très contestée jusqu'à une date assez récente, elle est aujourd'hui admise par la grande majorité des paléontologues et des biologistes.

Les idées évolutionnistes de Darwin diffusent rapidement, mais non sans controverses, à travers l'Europe. En Allemagne, le propagateur de ces idées est le biologiste Ernst Hæckel (1834-1919). Ce dernier publie d'ailleurs en 1866 le premier arbre montrant la filiation de l'ensemble des organismes vivants à partir d'une origine commune. Il forge, par ailleurs, un certain nombre de termes scientifiques comme les mots phylogénie, écologie, et on lui doit le principe de récapitulation: l'ontogénèse récapitule la phylogénèse. Formulé autrement, cela signifie que chaque organisme, au cours de son développement, ressemble successivement à tous ses ancêtres. Si cette vision des choses, excessive, est aujourd'hui abandonnée, le développement ontogénétique est souvent pris en compte pour l'identification de caractères permettant de décrire l'évolution, et utilisés pour les reconstructions phylogénétiques. Plus tard, en 1877, il publie un arbre généalogique de l'homme, allant même jusqu'à y inclure une espèce hypothétique, le pithécanthrope (littéralement le singe homme), intermédiaire entre les singes et les grands singes. Il est important de noter que dès lors, la classification devient prédictive. En se fondant sur les données connues, Hæckel postule l'existence d'un être vivant intermédiaire entre les singes et les hommes. C'est d'ailleurs à la lecture de son ouvrage qu'Eugène Dubois, médecin néerlandais, se rendra en Indonésie et finira par découvrir à Java des fossiles qu'il décrira en 1894 sous le nom de Pithecanthropus erectus (le singe homme érigé), connu aujourd'hui sous le nom d'Homo erectus.

26 Les paléontologues s'emparent eux aussi des idées darwiniennes et tentent de faire le lien entre les espèces fossiles et les espèces actuelles. En France, Albert Gaudry 
(1827-1908) publie en 1866, dans une étude consacrée aux animaux fossiles de Pikermi (Grèce), les cinq premiers arbres incluant à la fois des espèces fossiles et des espèces actuelles. Il y adjoint également dans sa figure sur l'évolution des proboscidiens une échelle de temps explicite indiquant le nom d'étages géologiques couvrant la partie supérieure du Néogène. Pour la première fois, les fossiles y sont explicitement considérés comme les ancêtres directs des formes actuelles. Cette vision des choses donnera naissance à la notion de groupe ancêtre : les amphibiens ancêtres des reptiles, par exemple - une idée perdurera jusqu'à des dates assez récentes et qui reste encore dans l'imaginaire collectif.

Charles Depéret (1854-1829) va lui s'attaquer à la reconstruction des lignées phylétiques :

L'évolution se fait avec une vitesse variable, mais par une très nombreuse série de rameaux, se développant parallèlement et par mutations graduelles à travers les étages géologiques, sans contacts ni passages d'un rameau à l'autre, sauf les cas de bifurcation qu'il nous est bien rarement donné de saisir avec certitude. (Depéret, 190)

Et il faut être très rigoureux :

Le moment est venu où l'évolution paléontologique doit être l'histoire de ce qui s'est réellement passé et non une image poétique de ce qui aurait pu se passer dans les temps anciens. (Depéret, 160).

Tous ces travaux conduisent à des classifications qui pouvaient différer entre elles selon les partis pris de leurs auteurs, mais presque tous les naturalistes s'accordaient à respecter les règles et les subdivisions instaurées par Linné et développées par ses successeurs. On se réfère toujours à ces différentes classifications comme faisant partie de la « classification classique ».

Il faut attendre la fin des années cinquante pour que les outils conceptuels permettant de définir une nouvelle classification, allant dans le sens du souhait de Darwin, soient enfin mis au point. Ce travail est l'œuvre de l'entomologiste allemand Willi Hennig (1950), qui proposera et développera une idée qui peut apparaître toute simple : il faut regrouper les organismes partageant des caractères évolués, au sein de groupes qu'il nomme monophylétiques, c'est-à-dire regroupant un ancêtre et tous ses descendants. Cette manière de considérer le problème met à mal certaines catégories de la classification classique. Les poissons, par exemple, n'ont plus de réalité classificatoire : on y trouvait, en effet, le dipneuste, qui possède un poumon, ce qui le rapproche davantage, d'un point de vue évolutif, des mammifères comme la vache plutôt que de la truite (Janvier et al., 1980). Il en va de même pour les reptiles, qui représentent ce que l'on nomme un grade, c'est-à-dire un stade évolutif : les oiseaux doivent être inclus dans les dinosaures car ils trouvent leur origine auprès d'un petit dinosaure carnivore et appartiennent donc au même groupe monophylétique - les exemples pourraient être nombreux. Ce bouleversement conceptuel induit une nouvelle manière de considérer les différents rapports entre les organismes : on ne cherche plus l'ancêtre ou le groupe ancêtre, mais le groupe frère, celui qui est le plus proche, celui qui partage un ancêtre commun direct. Par principe, l'ancêtre direct n'est pas connu, il devient un ancêtre commun hypothétique, mais dont on peut définir a posteriori les caractéristiques. La classification, jusque-là très figée, peut évoluer au gré des découvertes, ce qui ne va pas sans poser de problèmes de nomenclature. Ainsi, les travaux récents ont démontré que les plus proches parents (ou le groupe frère) des cétacés étaient les artiodactyles. En conséquence, il a fallu définir une nouvelle 
catégorie classificatoire, celle des Cétartiodactyles. Les catégories deviennent si nombreuses qu'il est parfois difficile de les maintenir dans le cadre étroit des subdivisions de la systématique linnéenne classique.

31 Ainsi, l'Origine des espèces, en faisant de la généalogie des organismes le fondement de la classification, a introduit de profonds bouleversements dans l'activité classificatoire. A travers elle, c'est toute notre manière de voir le monde qui a été transformée : l'Homme a quitté le sommet de l'arbre pour reprendre sa véritable place, en plein cœur du monde animal.

\section{BIBLIOGRAPHIE}

Anonyme, papyrus Ebers.

Albert le grand. De vegetalibus libri septem, historiae naturalis pars XVIII Editionem criticam ab Ernesto Meyero caeptam absolvit Carolus Jessen. 1415. Berlin, 1867.

Bauhin, Gaspard. Pinax Theatri Botanici sive Index in Theophrasti Dioscoridi Plinii et Botanicorum qui a seculo scripserunt, opera plantarum circiter sex millium ab ipsis exhibitarum nomina cum earundem Synonymiis a differentiis methodice secundum genera \& species proponens. 1623. 4 vol. Bâle : Regis, 1671.

Buffon, Georges Louis Leclerc, Comte de. Histoire Naturelle, t. 1, Théorie de la terre. Paris : Imprimerie Royale, 1749.

Cesalpino, Andrea. De plantis libri. 4 vol. Florence : Marescotti, 1583.

Colonna, Fabio. Aquatilium et terrestrium aliquot animalium, aliorumq. naturalium rerum observationes + Purpura hoc est de purpura ab animali testaceo fusa, de hoc ipso animali, aliisq. rarioribus testaceis quibus dam [inclut De Glossopetris dissertatio] [Ces textes constituent des appendices à : Minus cognitarum rariomque nostro cœelo orientium stirpium EKФРАГIC et à Minus cognitarum stirpium. Pars altera]. Rome : Jacobum Mascardum, 1616.

Cuvier, Georges. «Sur un nouveau rapprochement à établir entre les classes qui composent le Régne Animal ». Annales du Muséum d'Histoire Naturelle, 19 (1812): 73-84.

---. Discours sur les révolutions du globe et sur les changements qu'elles ont produit dans le monde animal. Paris : Dufour et d'Ocagne, 1825.

Darwin, Charles. On the Origin of Species by Means of Natural Selection, or the Preservation of favoured races in the struggle for life. London: John Murray, 1859.

---. On the Origin of Species by Means of Natural Selection, or the Preservation of Favoured Races in the Struggle for Life. 4th edition. London: John Murray, 1866.

---. The Origin of Species by Means of Natural Selection, or the Preservation of Favoured Races in the Struggle for Life. 5th edition. London: John Murray, 1869.

Depéret, Charles. Les Transformations du monde animal. Paris : Flammarion, 1907.

Dioscoride. « Materia medica ». Lyon : Antoine Vincent, 1554. 
Dubois, Eugène. Pithecanthropus Erectus: eine menschnaehnliche Uebergangsform aus Java. Landesdruckerei : Batavia, 1894.

Gaudry, Albert. Considérations générales sur les animaux fossiles de Pikermi. Paris : Savy, 1866. Gesner, Conrad. Historia animalium, livre I (De quadrupedibus uiuiparis). Zurich 1551.

Hæckel, Ernst. Generelle Morphologie der Organismen. Berlin : Reimer, 1866.

---. Anthropogenie ; oder, Entwickelungsgeschichte des menschen, Keimes- und stammesgeschichte. Leipzig : Engelmann, 1877.

Hennig, Willi. Grundzüge einer Theorie der phylogenetischen Systematik. Berlin : Deutscher Zentralverlag, 1950.

Hooke, Robert. Lectures and Discourses of Earthquakes and Subterraneous Eruptions. London: Waller, 1668.

Huxley, Thomas. « Remarks upon Archaeopteryx lithographica ». Proceedings of the Royal Society, 16 (1868): 243-48.

Janvier, Philippe, Tassy Pascal, Thomas Herbert. « Le cladisme ». La Recherche, 117, (1980): 1396-1406.

Jussieu, Antoine-Laurent. Genera plantarum secundum ordines naturales disposita, juxta methodum in horto regio parisiensi extaram. Paris : Herissant et Barrois, 1789.

Lamarck, Jean Baptiste. Philosophie zoologique. Paris : Dentu, 1809.

Linné, Carl von. Genera plantarum eorumque characteres naturales secundum numerum, figuram, situm, \& proportionem omnium fructificationis partium. Leiden : Wishoff, 1737.

---. Species Plantarum, exhibentes plantas rite cognitas, ad genera relatas, cum differentiis specificis, nominibus trivialibus, synonymis selectis, locis natalibus, secundum systema sexuale digestas. Holmiae: Impensis Laurentii Salvii, 1753.

---. Systema Naturae: Per Regna Tria Naturae, Secundum Classes, Ordines, Genera, Species, cum characteribus, differentiis, synonymis, locis. Holmiae : Impensis Laurentii Salvii, 1758.

Magnol, Pierre. Prodromus historice generalis plantarum, in quo familice per tabulas disponuntur. Montpellier : Daniel Pech, 1689.

Meyer, H. von. Archaeopteryx litographica (Vogel-Feder) und Pterodactylus von Solenhofen. N. Jhb. Mineralogie, Geognosie, Geologie und Petrefakten-Kunde (1861), 678-79.

Orbigny, Alcide d'. Prodrome de paléontologie stratigraphique universelle des animaux mollusques et rayonnés. 3 vol. Paris : Masson, 1850-52.

---. Cours élémentaire de paléontologie et de géologie stratigraphique. Paris : Masson, 1840.

Owen, Richard. « On the Archeopteryx of Von Meyer, with a Description of the Fossil Remains of a Long-Tailed Species, from the Lithographic Stone of Solenhofen ». Philosophical Transactions of the Royal Society, 153 (1863): 33-47.

Palissy, Bernard. Recepte véritable par laquelle tous les hommes de la France pourront apprendre à multiplier et augmenter leurs thrésors. La Rochelle : Berton, 1563.

Pline l'ancien. Histoire Naturelle. 1469. Trad. Littré. 2 t. Paris : Dubochet, le Chevalier et comp., 1848-1850.

Ray, John. Historia plantarum: Species hactenus editas aliasque insuper multas noviter inventas \& descriptas complectens. 3 vol. London: Faithorne \& Kersey, 1686. 
---. Methodus plantarum emendata et aucta. ... Accedit Methodus graminum, juncorum et cyperorum specialis. Eodem auctore Londini [i.e. Amsterdam]: impensis Samuelis Smith \& Benjamini Walford. Et veneunt Amsteloedami apud Janssonio-Waasbergios, 1703.

---. Methodus plantarum nova. London: Faithorne \& Kersey, 1683.

Saint-Hilaire, Geoffroy. Philosophie anatomique. Paris : Baillière, 1822.

Scheuchzer, Johann Jakob. Physica sacra. 4 vol. Augsburg et Ulm : Pfeffel et Wagner, 1731.

Stenon, Nicolas. De solido intra solidum naturaliter contento dissertationis prodromus. Florence :

Typographia sub signa stellae, 1669.

Tassy, Pascal. « L'émergence du concept d'espèce fossile : le mastodonte américain (Proboscidea, Mammalia) entre clarté et confusion ». Geodiversitas 24 (2) (2002): 263-294.

Theophraste. Recherches sur les plantes. 5 vol. Paris : Les belles lettres, 2003-2006.

Tournefort, Joseph Pitton de. Élemens de botanique, ou méthode pour connoître les plantes. 3 vol. Paris : Imprimerie Royale, 1694.

Vinci, Léonard de. Codex Leicester. Manuscrit, 1508-1510.

\section{RÉSUMÉS}

Depuis l'antiquité, les hommes ont cherché à classer les espèces, manière de décrire le monde et de se l'approprier. A la suite de la systématisation de la nomenclature binominale par Carl von Linné (1758) et jusqu'aux premiers développements des théories évolutionnistes, la classification se veut être le reflet d'une échelle naturelle des êtres avec, bien entendu, une place prépondérante pour l'homme, classé chez les Primates (les premiers), tout au sommet de cette échelle. Avec la parution de L'Origine des espèces de Darwin, l'idée d'évolution, déjà dans l'air depuis les travaux de Lamarck notamment, amène à revoir les principes fondamentaux de la classification : d'une échelle traduisant la position respective des différentes espèces autour d'un monde fixe, créé par la volonté divine, il faut dorénavant y intégrer les fossiles et lire la classification dans une perspective temporelle. La classification doit devenir le reflet de l'histoire du vivant. Il est d'ailleurs symptomatique que la seule figure de L'Origine des espèces soit justement un modèle théorique de phylogénie, c'est-à-dire d'histoire évolutive. Avec cette illustration, les principes de base de la classification moderne sont jetés, mais il faudra attendre les années 1950 et le développement de la cladistique, sous l'impulsion de l'entomologiste allemand Willi Hennig, pour que le rêve de Darwin devienne une réalité.

Men have tried to classify species since time immemorial, classification being a means of describing the world and of appropriating it. From Carl von Linne's system of scientific classification and binominal nomenclature, to the first developments of evolutionary theories, classifications attempted to fashion a natural scale of living beings. Man was, of course, positioned with the Primates (i.e. the first ones) at the top of the tree. After the publication of Darwin's Origin of Species, the idea of evolution, which had stamped Lamarck's work (albeit as transformism), led scientists to imagine new ways of classifying the living. Moving away from a vision of classification as fixed and reflecting divine design, Darwin and his followers took fossils into account to redefine classification from a temporal point of view. Classification started then to represent the history of life. The fact that the only illustration in the Origin of Species is a theoretical phylogenetic tree is highly significant: the illustration set up the basis of modern classification, which was developed in the 1950s by Willi Hennig's phylogenetic systematics, making Darwin's dream come true. 
INDEX

Keywords : fossil, evolution, classification, systematics, Darwin

Mots-clés : fossile, évolution, classification, systématique, Darwin

\section{AUTEURS}

\section{FRANCIS DURANTHON}

Conservateur

Muséum d'Histoire Naturelle de Toulouse / LECP - Université Paul Sabatier

francis.duranthon@cict.fr 